\title{
Should assisted dying be legalised?
}

\author{
Thomas D G Frost ${ }^{1}$, Devan Sinha ${ }^{2}$ and Barnabas J Gilbert ${ }^{3 *}$
}

\begin{abstract}
When an individual facing intractable pain is given an estimate of a few months to live, does hastening death become a viable and legitimate alternative for willing patients? Has the time come for physicians to do away with the traditional notion of healthcare as maintaining or improving physical and mental health, and instead accept their own limitations by facilitating death when requested? The Universities of Oxford and Cambridge held the 2013 Varsity Medical Debate on the motion "This House Would Legalise Assisted Dying". This article summarises the key arguments developed over the course of the debate. We will explore how assisted dying can affect both the patient and doctor; the nature of consent and limits of autonomy; the effects on society; the viability of a proposed model; and, perhaps most importantly, the potential need for the practice within our current medico-legal framework.
\end{abstract}

Keywords: Assisted dying, Legalisation, Physician-assisted suicide, Euthanasia

\section{Introduction}

Over the past two centuries, the United Kingdom has experienced rapid population growth associated with a substantial decline in mortality from acute infectious diseases and poor nutrition [1]. As the average life expectancy has increased, so too have the rates of debilitating chronic illness - particularly coronary artery disease and cancers [2]. These diseases require years of treatment instead of the mere days to weeks that medicine once operated within [2]. Although healthcare systems have sought to adapt to such changes, aiming to prevent and treat such disease wherever possible, debate has arisen regarding those patients in the latter stages of chronic, incurable, terminal conditions [3,4]. Moreover, there is increasing recognition that the patient must be at the centre of health care decision-making, such that outcomes must be tailored to their individual needs and views. By extension, assisted dying might seem a logical step to help achieve these goals within the realm of endof-life decision making [5]. Several jurisdictions, notably Oregon (1997) and the Netherlands (2001) have already legalised assisted dying in some form. These factors have contributed to ongoing legislative discussions within Parliaments for almost a decade, with current opinion polling suggesting a majority of medical practitioners and the public in favour of physician-assisted suicide [6].

\footnotetext{
* Correspondence: Barnabas.gilbert@gtc.ox.ac.uk

${ }^{3}$ Green Templeton College, Woodstock Road, Oxford OX2 6HG, UK

Full list of author information is available at the end of the article
}

\section{Viability of assisted dying in practice}

In the UK, a model for assisted dying has been developed from the legal structure found within the Assisted Dying Bill introduced by Lord Falconer in the House of Lords in 2013 [7]. Assisted dying could only be considered under circumstances in which a patient of legal age is diagnosed with a progressive disease that is irreversible by treatment and is "reasonably expected to die within six months" [7]. Registered medical practitioners would make such decisions for patients with terminal illnesses. Addressing the technicalities of 'assisted dying' requires distinction between 'physician-assisted suicide' (offering patients medical actions or cessation of actions by which they can end their own life) and 'euthanasia' (whereby the medical practitioner actively induces death). In light of the strong hostility of the medical profession towards active euthanasia, this proposed model, as with previous attempts to legalise assisted dying, permitted only the former [8-10].

However, there is concern that such distinction may be unrealistic in practice because medical practitioners could find themselves with a patient who had failed to successfully end their own life and was subsequently left in a state of greater suffering. Were such a patient no longer able to give consent, a heavy burden would then be placed on the physician regarding how to proceed. Moreover, the practice of physician-assisted suicide might be deemed discriminatory, for example by giving only patients with good mobility control over their own method of death. 
The Assisted Dying Bill 2013 included the provision that any terminal prognosis must be confirmed and attested by a second registered practitioner. The strictness of such criteria has parallels to a similar doublephysician requirement when procuring a legal abortion under the 1967 Abortion Act. The stated aims of the provision in both cases are as follows: first, to check the accuracy of the prognosis upon which the decision was being made; second, to ensure that the situation meets the required criteria; and third, to check that such a decision was taken by the patient after full consideration of all available options $[11,12]$. By having a second independent doctor, the legislation ensures that all three checks are met without prejudice or mistake.

Problematic for any protocol for assisted dying is the fact that estimates of life expectancy in terminal prognoses are erroneous in $80.3 \%$ of cases [13]. Furthermore, the accuracy of such prognoses deteriorates with increased length of clinical predicted survival. Forecasts of survival times are based largely on past clinical experience, and the inherent variability between patients makes this more of an art than a science. This brings to concern both the accuracy of any prognosis meeting the six-month threshold and the validity of requests for assisted dying based partly or wholly on predicted survival times. Whilst the majority of errors in life expectancy forecasts are a matter of over-optimism and hence would not affect either of those two concerns, many cases remain unaccounted for. Overly pessimistic forecasts occur in $17.3 \%$ of prognoses; hence we must decide whether the one in six patients making a decision based on an inaccurate prognosis is too high a cost to justify the use of this system. Patients requesting an assisted death often cite future expectations of dependency, loss of dignity, or pain [14]. If the hypothetical point at which the progression of their illness means they would consider life to be not worth living is not, as informed, mere weeks away but in fact many more months, then this information would have resulted in a different decision outcome and potentiated unnecessary loss of life.

Whilst the presence of a second doctor would be expected to mitigate such forecasting errors, the anchoring bias of the initial prediction may be enough to similarly reduce the accuracy of the second estimate. It is prudent to question the true independence of a second medical practitioner, and whether this second consultation could become more of a formality, as has now become the case with abortion [15].

Another challenge for an assisted dying system would be to recognise whether patients requesting death were legally competent to make that decision. Consider that any request for suicide from a patient with clinical depression is generally categorised as a manifestation of that mental disorder, thereby lacking capacity. It is arguably impossible to separate out the natural reactions to terminal illness and clinical depression. Indeed, there is evidence that major depressive disorders afflict between $25 \%$ and $77 \%$ of patients with terminal illness $[16,17]$. Any protocol for assisted dying must first determine what qualifies as a 'fit mental state' for a terminal patient.

\section{The need for assisted dying}

It could be argued that a doctor's fundamental duty is to alleviate forms of suffering in the best interests of the patient. The avoidance of physical pain, as an obvious manifestation of suffering, might explain why assisted dying would be both necessary and within the duties of a doctor to provide. The evolving principle in common law known as the 'Doctrine of Double Effect' offers a solution to this problem [18]. This legal judgement stated that "[a doctor] is entitled to do all that is proper and necessary to relieve pain even if the measures he takes may incidentally shorten life". This entails that a protocol already exists for patients searching for an escape from chronic pain. Furthermore, numerous retrospective studies have revealed very little correlation between opioid dose and mean survival times: one study of over 700 opioid-treated patients found that the variation in survival time from high-dose opioid treatment is less than $10 \%$ [19-21]. It can therefore be said that pain alone, if appropriately managed, should never be cause for considering assisted dying as an alternative.

By contrast, the 'Doctrine of Double Effect' might be seen as a subjective interpretation that has been applied unequally due to a lack of specialist training or knowledge [22]. Despite this, the principle can be easily understood and poor awareness can be remedied by improvements in medical education and standardisation of protocols. Moreover, should we choose to accept arguments for assisted dying that are based upon inadequate administration of pain medication, we set a precedent for conceding shortcomings in palliative care and other end-of-life treatments. Offering hastened death could become an alternative to actively seeking to improve such failings.

Whilst much has been made of the 'pain argument' here, the call for assisted dying is rarely this simple. Many patients also suffer a loss of dignity, often due to their lack of mobility - the inability to relieve oneself without help is a potent example. Beyond this are additional fears of further debilitation and the emotional costs of dealing with chronic illness, both for the patient and for their relatives and friends. A study of terminal patients in Oregon showed that these were the most significant reasons behind requests for assisted suicide, the next commonest reason being the perception of themselves as a 'burden' [14]. Clearly, we could seek to 
provide balanced, compassionate medical care for these patients, and still fail to address these points.

Developments in healthcare and technology may reduce this emotional burden, but remain an imperfect solution.

\section{Rights of patients and limitations of their autonomy}

J. S. Mill's pithy dictum describes autonomy as follows: "over himself, over his own body and mind, the individual is sovereign" [23]. Not only has the sanctity of bodily autonomy profoundly influenced the development of liberal democracies, it has also provoked a holistic shift in making our healthcare systems more patient-centred "care that meets and responds to patients' wants, needs and preferences and where patients are autonomous and able to decide for themselves" [5]. The ethical principle of controlling the fate of one's own body is inherently relevant to the debate on assisted dying. It is difficult to reconcile that citizens may have the right to do almost anything to and with their own bodies- from participating in extreme sports to having elective plastic surgery yet a terminal patient cannot choose to avoid experiencing additional months of discomfort or loss of dignity in their final months of life.

Expectation of individual liberty has been codified in law. The right to bodily autonomy has been interpreted to be included under Article 8 - the right to privacy - of the European Convention on Human Rights (ECHR) and subsequently the Human Rights Act (HRA) [24,25]. Moreover, the ECHR underpins the right of individuals to 'inherent dignity' [26]. Hence, if an individual feels that dignity is unattainable due to the progression of a terminal illness, then taking recourse though assisted dying ought to be a legitimate option.

Conversely, there are two notable oversights in this interpretation of a right to assisted dying as an extension of the principles of bodily autonomy:

First, it would be wrong to view individual liberty as absolute. The HRA allows for exceptions to Article 8 on grounds of 'health or morals' [25]. The principle of autonomy is not inviolable. Governments have limited such privileges for the protection of individuals and society, for example by criminalizing the use of recreational drugs or the selling of one's own organs. The preservation of life by denying assisted dying could fall within this category.

Second, the right of autonomy is not necessarily intrinsic to human beings but, as Kant argued, is dependent on our 'rational nature' [27]. This concept sees autonomy as an exercise of 'evaluative choice' [27], requiring rationality on the part of individuals to appreciate the nature of options and their consequences. To achieve true autonomy, there must be sufficient information to make those rational decisions; this is the basis of informed consent and why it is a fundamental duty of a doctor to offer a patient an informed series of treatment options [28]. The logistical issue is that doctors are unable to advise patients regarding the point at which their situation becomes less preferable to being dead. No doctor (or individual) has any knowledge or experience of what 'death' may be like. Hence, in this case, the idea of exercising true autonomy through informed consent might be considered meaningless.

Legalising assisted dying by attempting to establish an absolute right to bodily autonomy may undermine other individual and group rights. Vulnerable patients may feel pressured into assisted dying because of social, emotional, or financial strains placed on family and/or friends. This is exemplified by the trend showing that the proportion of patients stating 'relief of burden' on others as the reason for requesting assisted dying has risen from $17 \%$ to $25 \%$ in Oregon since legalisation [29]. One could even consider the risk of assisted dying becoming an expected choice rather than a free one. Thus, assisted dying may erode the elemental right to life of terminal patients as the value of their life becomes tied to relative costs to society and to those around them.

Moreover, by creating one class of individuals for whom life is expendable, that particular view may be extended by society to all groups possessing such attributes (e.g. the permanently disabled). There would be a definite risk to the rights of these vulnerable groups in the form of society being less willing to provide for their health and social care.

It is often raised that the limited legalisation of assisted dying would inevitably become extended in scope, but this is not necessarily a flaw. Even if the right to determine the manner of death were later extended to a wider group of people, posterity may reflect positively on such a change, just as extending the franchise to women ultimately led to legislation demanding equal pay.

\section{Effect on health professionals and their role}

'To act in the best interest of the patient' is often cited as a central duty of the doctor [28]. This concept of 'best interest' guiding the doctor's action has seen the development of two important ethical principles: beneficence and non-maleficence. Beneficence mandates that the actions of the doctor must be aimed to bring about benefit (clinical improvement) for the patient, usually measured in terms of reduced morbidity or mortality; non-maleficence requires that the doctor not carry out treatment that is likely to cause overall harm the patient [30]. These traditional ethical imperatives on a doctor both conflict with intentionally hastening the death of a patient, and a resolution of this tension would require redefining what constitutes 'acting in the best interest'. 
A further dimension is the potential reluctance of health professionals to engage in a practice that contravenes their own ethical beliefs, particularly as this would affect doctors who never entered training in the knowledge that assisting patients to die would be an expected duty. This is certainly no argument against the introduction of assisted dying; indeed, a recent survey of a cohort of NHS doctors found that $46 \%$ would seriously consider requests from patients to undertake steps to hasten death [31]. It merely expresses the point that any early model would have to account for the fact that an initial $54 \%$ of the doctors in the NHS would be required to advise qualifying patients of assisted dying as a legitimate option, despite disagreeing with it in principle.

Furthermore, doctors who agree ethically with this practice may find themselves facing conflicts of interest. It is expensive to treat chronically ill patients, particularly in the final months of life [32]. Moreover, it would be difficult for commissioners to ignore the fact that the sustained treatment of one individual could deprive many others from access to surgery or access to novel drugs. Such an argument does not suggest that doctors or any other hospital staff would treat this practice without appropriate respect or care; rather it acknowledges the need for appropriate rationing of care and questions the intentions of service providers. The perception of an ulterior motive could negatively impact patient trust. One survey showed that a reasonable minority of patients $(27 \%)$ - and particularly particularly the elderly believe that legalising assisted dying would lessen their trust in their personal physician [33]. The costs of weakened trust in the doctor-patient relationship could far outweigh the benefits of assisted dying, particularly given the importance of trust when treating a chronic patient for an extended period of time.

\section{Conclusion}

There is no doubt that assisted dying would empower some patients to maximise control over the timing and manner of their own death. Such expression of autonomy would surely solidify moves towards a patientcentred approach to healthcare. However, the capacity for such consensual requests remains in doubt. Clinically, the patient's state of mind and the reliability of diagnostic predictions are of issue; philosophically, the idea of informed consent for death is contradictory. The implications for patients, physicians and society have been weighed extensively within this article. The central tenet throughout has been the balancing of an individual's right to escape a circumstance that they find intolerable, alongside the consequential changes to their other rights, and the rights and responsibilities of third parties. Ultimately, the challenge is for us as a society to decide where this balance lies.

\section{About the debate}

The Varsity Medical Debate was started in 2008 with the aim of allowing students, professors and members of the polis, to engage in discussion about ethics and policy within healthcare. Utilising the age-old rivalry between the two Universities, the debate encourages medical students from both Oxford and Cambridge to consider and articulate the arguments behind topics that will feature heavily in their future careers.

The debate was judged on the logic, coherence, and evidence in arguments, as well as flair in presentation. Although the debaters may not have necessarily agreed with their allocated side, the debate format required them to acknowledge a particular school of thought and present the key arguments behind it. Oxford, who opposed the motion, was awarded the victory in the debate; however, this does not mean that the judges believe that position ought to become public policy.

\section{Competing interests}

The authors declare that they have no competing interests.

\section{Authors' contributions}

All authors planned and elucidated the layout. TDGF and DS drafted the manuscript which was critically edited and added to by BJG. All authors have read and approved the final draft.

\author{
Acknowledgements \\ Debaters: \\ For Cambridge University: Hilmi Bayri (Trinity), Alistair Bolger (Jesus), Casey \\ Swerner (St Johns). \\ For Oxford University: Devan Sinha (Brasenose), Thomas Frost (Lincoln), Collis \\ Tahzib (Lincoln). \\ Convener: \\ Martin Farrell (Cambridge) \\ Judges: \\ Baroness Finlay: Professor of Palliative Care Medicine and former President of \\ the Royal Society of Medicine. \\ Dr. Roger Armour: Vascular Surgeon and Inventor of the Lens Free \\ Ophthalmoscope. \\ Mr. Robert Preston: Director of Living and Dying Well.
}

\section{Author details}

${ }^{1}$ Lincoln College, Turl Street, Oxford OX1 3DR, UK. ${ }^{2}$ Brasenose College, Oxford OX1 4AJ, UK. ${ }^{3}$ Green Templeton College, Woodstock Road, Oxford OX2 6HG, UK.

Received: 12 November 2013 Accepted: 11 January 2014

Published: 15 January 2014

\section{References}

1. Colgrove J: The McKeown thesis: a historical controversy and its enduring influence. Am J Public Health 2002, 92:725-729.

2. Yach D, Hawkes C, Linn Gould C, Hofman K: The global burden of chronic diseases: overcoming impediments to prevention and control. J Am Med Assoc 2004, 291(21):2616-2622. doi:10.1001/jama.291.21.2616.

3. Lankhorst EK, Spreeuwenberg C: Managing Chronic Conditions. Experience in Eight Countries. In European Observatory on Health Systems and Policies. Edited by Nolte E, Cécile K, Martin MK. The Netherlands: WHO Regional Office Europe; 2008.

4. Hudson PL, Kristjanson LJ, Ashby M, et al: Desire for hastened death in patients with advanced disease and the evidence base of clinical guidelines: a systematic review. Palliat Med 2006, 20:693-701.

5. Hogg C: Patient-Centred Care-Tomorrow's Doctors. GMC; 2004.

6. Harris D, Richard B, Khanna P: Assisted dying: the ongoing debate. Postgrad Med J 2006, 82(970):479-482. doi:10.1136/pgmj.2006.047530. 
7. House of Lords assisted Dying Bill: House of Lords. 2013. http://www. publications.parliament.uk/pa/bills/lbill/2013-2014/0024/2014024.pdf (accessed 23 October 2013).

8. House of Lords Assisted Dying for the Terminally III Bill: House of Lords. 2005. http://www.publications.parliament.uk/pa/ld200506/ldbills/036/ 2006036.pdf (accessed 15 Feb 2006).

9. Sanders K, Chaloner C: Voluntary euthanasia: ethical concepts and definitions. Nurs Stand 2007, 21(35):41-44.

10. Lee W, Price A, Rayner L, Hotopf M: Survey of doctors' opinions of the legalisation of physician assisted suicide. BMC Med Ethics 2009, 10:2.

11. House of Commons: Science and Technology Committee, Scientific Developments Relating to the Abortion Act 1967. Volume 1st edition. London: tso; 2007.

12. Royal College of Obstetricians and Gynecologists, Campaigns and Opinions. http://www.rcog.org.uk/what-we-do/campaigning-and-opinions/briefingsand-qas-/human-fertilisation-and-embryology-bill/brie-1.

13. Christakis NA, Lamont EB: Extent and determinants of error in physicians' prognoses in terminally ill patients. BMJ 2000, 320:469-473.

14. Ganzini L, Goy E, Dobscha S: Oregonians' reasons for requesting physician aid in dying. J Am Med Assoc Int Med 2009, 169(5):489-492. doi:10.1001/ archinternmed.2008.579.

15. Care Quality Commision. Findings of Termination of Pregnancy Inspections Published July 2012. http://www.cqc.org.uk/media/findings-terminationpregnancy-inspections-published.

16. Watson M, Lucas C, Hoy A, et al: Oxford Handbook of Palliative Care. Oxford: Oxford University Medical Press; 2005.

17. Fine R: Depression, anxiety, and delirium in the terminally ill patient. Proc (Bayl Univ Med Cent) 2001, 14(2):130-133.

18. R v Adams [1957] Crim LR 773.

19. Morita T, Tsunoda J, Inoue S, Chihara S: Effects of high dose opioids and sedatives on survival in terminally ill cancer patients. J Pain Symptom Manage 2001, 21(4):282-289. doi:10.1016/S0885-3924(01)00258-5.

20. Bengoechea I, Gutiérrez S, Vrotsou K, Onaindia M, Lopez J: Opioid use at the end of life and survival in a hospital at home unit. J Palliat Med 2010, 100828074323069. doi:10.1089/jpm.2010.0031

21. Thorns A, Sykes N: Opioid use in last week of life and implications for end-of-life decision-making. Lancet 2000, 356(9227):398-399. doi:10.1016/ S0140-6736(00)02534-4.

22. Preston T, Patterson J: The rule of double effect. N Engl J Med 1998, 338:1389-1391.

23. Mill JS: "On Liberty" in On Liberty and Other Essays. 14-15.

24. Human Rights Act. HMSO; 1998.

25. Judgment on the Merits Delivered by a Chamber. Y.F. v. TURKEY, no. 24209/94, ECHR 2003-IV

26. McCrudden C: Human dignity and judicial interpretation of human rights. Eur J Int Law 2008, 19(4):655-724. doi:10.1093/ejil/chn043.

27. Savulescu J: Autonomy, the Good Life and Controversial Choices. 2006.

28. General Medical Council: Good Medical Practice. GMC; 2013.

29. Oregon Department of Human Services. Fifth Annual Report on Oregon's Death with Dignity act. http://egov.oregon.gov/DHS/ph/pas/docs/year5.pdf.

30. Campbell, et al: Medical Ethics. Oxford: Oxford University Press; 1997.

31. Ward B, Tate P: Attitudes among NHS doctors to requests for euthanasia. BMJ 1994, 308:1332.

32. National Audit Office. End of Life Care: Report by the Comptroller and Auditor General. HC 1043 Session 2007-2008. NAO; 2008.

33. Hall $M$, Trachtenberg M, Duggan E: The impact on patient trust of legalising physician aid in dying. J Med Ethics 2005, 31(12):693-697. doi:10.1136/jme.2004.011452.

doi:10.1186/1747-5341-9-3

Cite this article as: Frost et al: Should assisted dying be legalised?

Philosophy, Ethics, and Humanities in Medicine 2014 9:3.

\section{Submit your next manuscript to BioMed Central and take full advantage of:}

- Convenient online submission

- Thorough peer review

- No space constraints or color figure charges

- Immediate publication on acceptance

- Inclusion in PubMed, CAS, Scopus and Google Scholar

- Research which is freely available for redistribution 